\title{
Os efeitos do programa de exercícios físicos start sobre os níveis de aptidão física para a saúde em escolares adolescentes com indicadores de risco a saúde
}

\section{The effects of the program of physical exercises start over the levels of physical fitness for health in schoolchildren with indicators of health risk}

\author{
Monique C. Albuquerque ${ }^{1 *}$, Bruno Rafael C. Costa ${ }^{1}$, Ewerton R. Mafra ${ }^{1}$, Jhon Bruce M. \\ Santos ${ }^{1}$, Shelry S. Silva ${ }^{1}$
}

ARTIGO ORIGINAL | ORIGINAL ARTICLE

\begin{abstract}
Dentre os fatores associados à obesidade, reconhecemos a aptidão física para a saúde (ApFS) como um fator que se relaciona ao aumento ou à diminuição da obesidade. No intuito de melhorar as ApFS, criamos o programa de exercício físico START. O objetivo foi verificar os efeitos do programa sobre os níveis de ApFS enfatizando o IMC, RCQ e resistência abdominal de adolescentes matriculados no colégio Amazonense Dom Pedro II. A amostra contou com 63 escolares com média de idade de 16,11 anos, sendo 16 do grupo experimental (GE) e 47 do grupo controle (GC). Para a recolha e avaliação dos dados, utilizou-se o sistema PROESP-Br validado por (GAYA et al., 2016). Resultados: Houve diferença estatisticamente significativa entre pré e pós-intervenção para + GE 17 anos na RCQ $(p=0.03) e$; + GE 17 anos no Sit-up $(p=0.02)$; para $\delta \mathrm{GC} 17$ anos na RCQ $(p=0.04)$, đGE 16 anos na RCQ $e$ no Sit-up $(p=0.00, p=0.00) e$; $\mathrm{GE} 17$ anos na RCQ e no Sit-up $(p=0.02, p=0.00)$. Os resultados obtidos no programa START apontam que intervenções com 21 sessões e frequência de 3 vezes semanais podem gerar mudanças significativas nos níveis de ApFS. Palavras-chave: PROESP-Br, intervenção, aptidão física, adolescentes.
\end{abstract}

ABSTRACT

Among the factors associated with obesity, we recognize the physical activity for health (PAFH) as a factor that relates to the increase or reduction of obesity. In order to improve the physical activity for health, we created the physical exercise program START. The objective was to verify the effects of the program on the levels of physical activities for health, emphasizing the BMI, WHR and abdominal strength of adolescents enrolled in college Amazonense Dom Pedro II. The sample consisted of 63 schoolchildren with an average age of 16,11 years, being 16 in the experimental group (EG) and 47 in the control group (CG). For the collection and evaluation of data, we used the system PROESP-BR validated by (GAYA et al., 2016). Results: There was a statistically significant difference between pre- and post-intervention for $q$ GE 17 years on the WHR ( $p=0,039)$; and $q$ GE 17 years in the sit-up ( $p=0,029)$; for $\delta$ GC 17 years on the WHR ( $\mathrm{p}=0,045)$, $\delta$ GE 16 years on the WHR and sit-up $(\mathrm{p}=0,005, \mathrm{p}=0,000)$; and $\delta$ GE 17 years on the WHR and sit-up $(p=0,024, p=0,009)$. The results obtained in the program START point out to intervention with 21 sessions, and frequency of 3 times a week, can generate significant changes in the levels of pafh.

Keywords: PROESP-Br, intervention, physical fitness, adolescents.

\footnotetext{
${ }^{1}$ Centro Universitário Fametro, Manaus-AM, Brasil

* Autor correspondente: moniquecunha88@gmail.com
} 


\section{INTRODUÇÃo}

Considerando que o número de crianças e jovens obesos aumentou 10 vezes nas últimas quatro décadas, o Imperial College London em parceria com a Organização mundial de Saúde (OMS, 2017), lideraram um estudo epidemiologico com 31, 5 milhões de pessoas entre os 5 e os 19 anos, e estimam que em 2022 o número obesos seja ainda maior. A obesidade infato-juvenil tem sido tema de diversas pesquisas e é caracterizada como uma doença complexa e de etiologia multifatorial, conforme Bucco e Zubiaur-Gonzáles (2013), Espínola et al., (2014). Dentre os fatores que promovem a obesidade, reconhecemos a aptidão fisica para a saúde (ApFS) como um fator que se relaciona ao aumento ou à dimnuição da obesidade em um contexto sinérgico, conforme Stodden e Goodway (2007). Diante do exposto, torna-se emergente a tomada de decisões práticas e conscientes de profissionais da educação e da saúde, promovendo a mudança de hábitos no que tange o aumento da prática de atividade física, a melhora dos níveis de ApFS e o aumento da expectativa de vida saudável. Nesse contexto, entende-se que a escola situa-se no centro das preocupações com a educação para a saúde, de acordo com Marques e Gaya (1999). No intuito de incentivar um ambiente escolar de caractetisticas leptogênicas, criamos o programa START, uma proposta extra-curricular de exercícios físicos voltados para a promoção os hábitos saudáveis e para a melhora dos níveis de ApFS. O objetivo desse estudo foi verificar os efeitos do programa START sobre os níveis de ApFS enfatizando os aspectos morfológicos (índice de massa corporal, relacão cintura/quadril) e aspecto funcional (resistência abdominal) de adolescentes com média de idade 16.11 nos matriculados numa escola da rede de ensino público na cidade Manaus.

\section{MÉTODO}

Este é um estudo de caráter experimental, com intervenção controlada por meio do programa de exercício físico START que foi desenvolvido durante 2 meses, totalizando 21 sessões distribuídas em 7 semanas, sendo 3 sessões/semana - em dias alternados - com a duração de $50 \mathrm{~min} / \mathrm{sessão,} \mathrm{realizadas} \mathrm{no} \mathrm{horário}$ de 11:30 às 12:20. As sessões foram compostas por exercícios de contração isométrica e isotônica, a exemplo temos (pranchas lateral, básica, alta, em 3 apoios, em dois apoios com cadeia cruzada, com elevação de braços, com rotação de tronco, entre outras).

\section{Participantes}

A amostra foi composta por 63 escolares adolescentes com idade compreendida entre $14 \mathrm{e}$ 17 anos, de ambos os sexos, matriculados no nível médio do ensino regular - Escola Estadual Dom Pedro II - localizada na zona centro-sul da cidade de Manaus-AM. Os mesmos foram separados em dois grupos, onde 22 participaram voluntarimente do grupo experimental (GE) e 41 no grupo controle (GC). Foram incluídos na amostra adolescentes que apresentaram, no mínimo, 2 indicadores de risco à saúde, no que se refere às ApFS, conforme os pontos de corte estratificados por idade e sexo estabelecidos pelo sistema PROESP-Br (2016). Os adolescentes e seus pais/responsáveis assinaram o termo de consentimento livre esclarecido (TCLE), submetido à Plataforma Brasil e homologado pelo CEP 5015 - Centro Universitário Nilton Lins, de modo a garantir o anonimato e confidencialidade dos dados

\section{Instrumentos e Procedimentos}

Para a recolha de dados e avaliação da amostra, utilizou-se a versão atualizada do sistema PROESP-BR validado por (GAYA et al., 2016). Optou-se por dar ênfase nos indicadores de ApFS, incluindo os testes morfológicos e funcionais: índice de massa corporal (IMC), para estimar o excesso de peso; razão cintura estatura, para estimar o excesso da gordura visceral e; número de abdominais em 1 minuto (Sit-up), para estimar a resistência muscular localizada. Este sistema permite a avaliação dos adolescentes numa escala categórica de dois graus, sendo eles zona de risco à saúde (ZRS) e zona saudável (ZS) conforme os pontos de corte estratificados por idade e sexo. Para mais detalhes consultar (Gaya et al., 2016). O processo 
de recolha de dados ocorreu, tanto para GC quanto para GE, em dois momentos distintos definidos como pré-intervenção e pósintervenção.

\section{Análise estatística}

Para análise estatística utilizou-se o programa IBM-SPSS versão 23.0. Foi feita a comparação entre os momentos de pré e pós-intervenção para GC e GE por meio teste paramétrico $t$ para medidas emparelhadas (Paried-Sample $t$ Test) com $p<0,05$ como valor de significância com intervalo de confiança $95 \%$.

\section{RESULTADOS}

Apresentaremos os resultados da análise descritiva e comparativa dos testes morfológicos e funcionais da ApFS entre os momentos de pré e pós-intervenção do programa START para grupos controle e experimental feminino ( $+\mathrm{GC}$ ) e ( $(\mathrm{GE})$ e; grupos controle e experimental

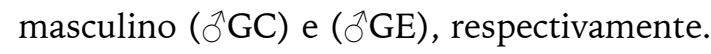

Conforme os dados expostos na tabela 01 verificou-se que não houve mudanças significativas na comparação entre os momentos pré e pós-intervenção para o †GC. As médias dos testes, tanto no pré quanto no pós-intervenção, classificam o $\odot$ GC na ZRS conforme os pontos de corte do PROESP-Br (2016). No entanto, para o †GE, verificou-se a evolução e transição da ZRS (pré-intervenção) para a ZS (pós-intervenção), tendo esse grupo reduzido significativamente $(p=0.03)$ a média da circunferência abdominal de $75.3 \mathrm{~cm}$ para $71.0 \mathrm{~cm} \mathrm{e;} \mathrm{aumentado}$ significativamente $(p=0.02)$ a quantidade média de abdominais no sit-up de 25.0 repetições para 41.6 repetições. Não verificou-se mudanças significativas para o IMC de ambos os grupos, no entanto percebeu-se uma tendência do GE em diminuir o IMC após a intervenção.

Tabela 1

Dados descritivos e comparativos dos testes morfológicos e funcionais de ApFS entre os momentos pré e pós-intervenção para o $+G C$ e $q G E$

\begin{tabular}{|c|c|c|c|c|c|c|}
\hline & \multirow{3}{*}{ Variáveis } & & \multirow{2}{*}{\multicolumn{2}{|c|}{$\begin{array}{c}\text { q GC }(=24) \\
\text { Média } \pm \text { D.P }\end{array}$}} & \multirow{2}{*}{\multicolumn{2}{|c|}{$\frac{q \text { GE }(n=10)}{\text { Média } \pm \text { D.P }}$}} \\
\hline & & & & & & \\
\hline & & & $\begin{array}{c}\text { Pré- } \\
\text { intervenção }\end{array}$ & $\begin{array}{c}\text { Pós- } \\
\text { intervenção }\end{array}$ & $\begin{array}{c}\text { Pré- } \\
\text { intervenção }\end{array}$ & $\begin{array}{c}\text { Pós- } \\
\text { intervenção }\end{array}$ \\
\hline \multirow{9}{*}{ 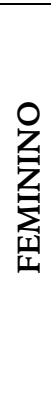 } & \multirow{3}{*}{$\operatorname{IMC}\left(\mathrm{Kg} / \mathrm{m}^{2}\right)$} & 15 anos & $30,7 \pm 5.68^{a}$ & $30.78 \pm 5.68^{a}$ & $Q$ & $Q$ \\
\hline & & 16 anos & $29.9 \pm 5.26^{\mathrm{a}}$ & $29.90 \pm 5.26^{a}$ & $Q$ & $Q$ \\
\hline & & 17 anos & $29.1 \pm 4.43^{\mathrm{a}}$ & $29.2 \pm 4.39^{\mathrm{a}}$ & $25.23 \pm 2.10^{\mathrm{a}}$ & $24.56 \pm 1.61^{\mathrm{b}}$ \\
\hline & \multirow{3}{*}{$\operatorname{RCQ}(\mathrm{cm})$} & 15 anos & $88.4 \pm 12.93^{a}$ & $88.5 \pm 12.76^{a}$ & $Q$ & $Q$ \\
\hline & & 16 anos & $86.9 \pm 9.01^{a}$ & $87.6 \pm 9.04^{\mathrm{a}}$ & $Q$ & $Q$ \\
\hline & & 17 anos & $84.7 \pm 11.27^{\mathrm{a}}$ & $85.0 \pm 11.45^{\mathrm{a}}$ & $75.3 \pm 8.08^{b}$ & $71.0 \pm 7.00^{\mathrm{b} *}$ \\
\hline & \multirow{3}{*}{$\begin{array}{l}\text { Número de } \\
\text { abdominais em } 1 \\
\text { minuto (Sit-up) }\end{array}$} & 15 anos & $18.7 \pm 12.82^{\mathrm{a}}$ & $19.0 \pm 11.79^{a}$ & $Q$ & $Q$ \\
\hline & & 16 anos & $22.0 \pm 11.47^{a}$ & $20.0 \pm 8.61^{a}$ & $Q$ & $Q$ \\
\hline & & 17 anos & $23.3 \pm 9.08^{b}$ & $22.0 \pm 7.79^{a}$ & $25.0 \pm 7.02^{b}$ & $41.6 \pm 3.05^{\mathrm{b} *}$ \\
\hline
\end{tabular}

Na tabela 02 , ao comparar as médias do $\partial^{7} \mathrm{GC}$ , verificou-se aumento significativo $(p=0.04) \mathrm{da}$ média da circunferência abdominal de $87.0 \mathrm{~cm}$ para $88.7 \mathrm{~cm}$, indicando valores críticos de risco à saúde, classificando-os na ZRS conforme os pontos de corte do PROESP-Br (2016). No entanto, as mudanças que ocorreram após a intervenção para o $\widehat{\jmath}$ GE evidenciam os benefícios do programa START com a redução significativa $(p=0.03)$ da média da circunferência abdominal de $80.8 \mathrm{~cm}$ para $77.6 \mathrm{~cm}$ para 16 anos e; de 84.0 $\mathrm{cm}$ para $81.0 \mathrm{~cm}$ para 17 anos, bem como o aumento significativo $(p=0.02)$ da quantidade média de abdominais no sit-up de 28.0 repetições para 49.6 repetições para 16 anos e; de 27.0 repetições para 47.5 repetições para 17 anos. As médias do $\widehat{T}$ GE apresentadas após a participação no programa START indicam a evolução do ${ }^{\top} \mathrm{GE}$ da ZRS para a ZS, conforme os pontos de corte do PROESP-Br (2016). 
Tabela 2

Dados descritivos e comparativos dos testes morfológicos e funcionais de ApFS entre os momentos pré e pós-intervenção para o

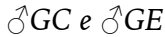

\begin{tabular}{|c|c|c|c|c|c|c|}
\hline & \multirow{3}{*}{ Variáveis } & & \multirow{2}{*}{\multicolumn{2}{|c|}{$\begin{array}{c}\text { JGC }(\mathrm{n}=17) \\
\text { Média } \pm \text { D.P }\end{array}$}} & \multirow{2}{*}{\multicolumn{2}{|c|}{$\begin{array}{c}\text { Média } \pm \text { D.P } \\
\text { Mé } 12)\end{array}$}} \\
\hline & & & & & & \\
\hline & & & $\begin{array}{c}\text { Pré- } \\
\text { intervenção }\end{array}$ & $\begin{array}{c}\text { Pós- } \\
\text { intervenção }\end{array}$ & $\begin{array}{c}\text { Pré- } \\
\text { intervenção }\end{array}$ & Pós-intervenção \\
\hline \multirow{9}{*}{ 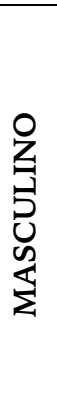 } & \multirow{3}{*}{$\operatorname{IMC}\left(K g / m^{2}\right)$} & 15 anos & $26.7 \pm 2.07^{\mathrm{a}}$ & $26.8 \pm 1.94^{\mathrm{a}}$ & $Q$ & $Q$ \\
\hline & & 16 anos & $27.9 \pm 3.11^{\mathrm{a}}$ & $28.08 \pm 3.10^{a}$ & $25.4 \pm 0.78^{a}$ & $25.0 \pm 0,82^{a}$ \\
\hline & & 17 anos & $28.5 \pm 4.15^{\mathrm{a}}$ & $28.8 \pm 4.07^{\mathrm{a}}$ & $25.9 \pm 0.61^{b}$ & $25.4 \pm 0,82^{\mathrm{b}}$ \\
\hline & \multirow{3}{*}{$\operatorname{RCQ}(\mathrm{cm})$} & 15 anos & $89.5 \pm 4.79^{\mathrm{a}}$ & $90.0 \pm 4.76^{a}$ & $Q$ & $Q$ \\
\hline & & 16 anos & $85.7 \pm 12.05^{\mathrm{a}}$ & $86.3 \pm 12.62^{\mathrm{a}}$ & $80.8 \pm 1.51^{\mathrm{a}}$ & $77.6 \pm 1.51^{\mathrm{b} *}$ \\
\hline & & 17 anos & $87.0 \pm 14.53^{\mathrm{a}}$ & $88.7 \pm 15.25^{a *}$ & $84.0 \pm 4.96^{\mathrm{a}}$ & $81.0 \pm 5.03^{\mathrm{b} *}$ \\
\hline & \multirow{3}{*}{$\begin{array}{l}\text { Número de } \\
\text { abdominais em } 1 \\
\text { minuto (Sit-up) }\end{array}$} & 15 anos & $37.7 \pm 11.23^{\mathrm{b}}$ & $37.7 \pm 9.74^{a}$ & $Q$ & $Q$ \\
\hline & & 16 anos & $35.3 \pm 7.81^{\mathrm{a}}$ & $35.1 \pm 7.35^{\mathrm{a}}$ & $28.0 \pm 5.56^{\mathrm{a}}$ & $49.6 \pm 3.64^{\mathrm{b} *}$ \\
\hline & & 17 anos & $32.8 \pm 5.69^{a}$ & $32.7 \pm 7.43^{a}$ & $27.0 \pm 5.03^{\mathrm{a}}$ & $47.5 \pm 1.29^{\mathrm{b} *}$ \\
\hline
\end{tabular}

\section{DISCUSSÃO}

Os resultados apresentados nessa pesquisa revelam que o programa de intervenção de atividade física extra-curricular START, teve efeito positivo na mudança dos níveis de ApFS enfatizando os aspectos morfológico RCQ e funcional (resistência abdominal) para ambos os sexos. As mudanças ocorridas após a intervenção para GE, demonstraram a transição dos adolescentes entre a ZRS e a a ZS conforme os pontos de corte estratificados pelo sistema PROESP-Br (2016). Apesar de não ter ocorrido mudanças significativas no IMC, percebeu-se uma tendência para a redução dessa medida conforme o exposição à prática de exercícios físicos cem frequência.

As mudanças decorrentes da prática de atividade física evidenciadas nesse estudo corroboram com vários programas de intervenção para escolares adolescentes, confome NeumarkSztainer et al. (2003) que atingiram mudanças nos indicadores de ApFS após 16 semanas (5x/semana); Silva et al. (2012) que atigiram mudanças significativas nos níveis de ApFS após 16 semanas de intervenção. Com os mesmos propósitos, Cardoso et al. (2014); Marques (2014) com um programa de intervenção de 30 aulas (2x/semana) alcançaram mudanças significativas nos níveis de flexibilidade e resistência abdominal e; Bianchini (2016) que melhorou os níveis de ApFS em 16 semanas (3x/semena) de intervenção.
Quanto a variável IMC, não verificou-se mudanças significativas na comparação entre os momentos pré e pós-intervenção. Uma provável justificativa para esse fato refere-se a ausência do acompanhamento nutricional, podendo caracterizar-se como uma limitação dessa pesquisa. Sobre esse item, diversas pesquisas apontam maior tendência para a mudança no IMC quando o programa de intervenção é direcionado à prática de exercício físico associado ao companhamento, controle e orientação nutricional. (Graf et al., 2005; Vasques, 2012; Edwards, 2015; Foster et al,. 2018)

Diante do exposto torna-se notório a necessidade de mais programas de intervenção no ambinte escolar, no que se refere a promoção da melhoria das condições de saúde dos escolares bem como dos hábitos saudáveis, em conformidade com os estudos diagnósticos dos nívies de ApFS (Sousa e Costa, 2017; Guedes et al., 2010; Spohr et al., 2014; Nogueira et al., 2014).

\section{CONCLUSÕES}

Os Os resultados obtidos no progrma START apontam que interveções com 21 semanas e frequência, executadas 3 vezes por semana, podem gerar mudanças significativas nos níveis de aptidão física para a saúde em adolescentes de ambos os sexos. Todavia, para que os resultados sejam mais expressivos, deve-se associar a prática de exercício físico à dieta nutricional balanceada. 
Agradecimentos:

Nada a declarar.

\section{Conflito de Interesses:}

Nada a declarar.

\section{Financiamento:}

Nada a declarar.

\section{REFERÊNCIAS}

Bucco L., \& Zabiaur-González, M. (2013). Desarollo de las habilidades motoras fundamentales em función del sexo y del índice de massa corporal em escolares [Versão eletrônica]. Caderno de Psicología del Deporte, 13(2), 63-72.

Espínola, J. A., Capristano, R., Alexandre, J. M., Silva, J., Beltrame, T. S. (2014) Efeito dos exercícios físicos na aptidão física de crianças com sobrepeso. ConScientiae Saúde, 13(2), 281-288. doi:10.5585/ConsSaude.v13n2.4682

Imperial College London e Organização mundial de Saúde (2017). Worldwide trands in body-mass index, underweight, overweitght, and obesity from 1975 to 2016: a pooled analysis os 2416 population-based measurement studies in 128.9 miolion children, adolescents, and adults. NCD Risk Factor Collaboration (NCD-RisC), 390(10113), 2627-2642. doi: http://dx.doi.org/10.1016/ S0140-6736(17)32129-3.

Graf, C., Rost, S. V., Koch, B., Heinen, S., Falkowski, G., Dordel, S., et al. (2005). Data from the StEP TWO programme showing the effect on blood pressure and different parameters for obesity in overweight and obese primary school children. Cardiology Young, 15(3), 291-298.

Foster, G. D., Sherman, S., Borradaile, K. E., Grundy, K. M., Vander Veur, S. S., Nachmani, J., et al. (2008). A policy-based school intervention to prevent overweight and obesity. Pediatrics, 121 (4), e794-802.

Edwards, B. (2015). Childhood obesity: a school-based approach to increase nutritional knowledge and activity levels. Nursery Clinical North America, 40(4), 661-669.

Vasques, C. M. S. (2012). Atividade física em crianças com excesso de peso: Efeitos de um programa de intervenção.
Vila Real: Tese de doutoramento em Ciência do Desporto apresentada à Universidade de Trás-osMontes e Alto Douro.

Cardoso, M. A., Pereira, F. M., Afonso, M. R., Junior, I. C. R. (2014). Revista Brasileira Educação Física e Esporte, 28(1), 147-61.

Bianchini, J. A. A., Silva, D. F., Lopera, C. A., Antonini, V. D. S., Junior, N. N. (2016). Revista Brasileira Educação Física e Esporte, 30(4), 1051-1059.

Spohr, L. F., Fortes, M. O., Rombaldi, A. J., Halla, P. C., Azevedo, M. R. (2014). Revista Brasileira Atividade Física e Saúde, 19(3), 300-313.

Nogueira, J. A. D. \& Pereira, C. H. (2014). Aptidão Física relacionada à saúde de adolescentes participantes de programa esportivo. Revista Brasileira de Educação Física e Esporte, 28(1), 31-40.

Sousa, A. P. M \& Costa, J. M. M. (2017). Aplicação e avaliação do treinamento funcional na educação física escolar. Revista magsul de educação física na fronteira, 2(3), 21-33.

Stodden, D. F., \& Goodway, J. D. (2007). The dynamic association between motor skill development and physical activity. Joperd, 78(8), 33-49

Silva, D. F., Souza, L. L., Delfino, O. R.., Bianchini, A. A. J., Hintze, J. L., \& Junior, N. N. (2012). Efeitos de um programa multiprofissional de tratamento da obesidade e de sua cessação sobre a aptidão física relacionada à saúde de adolescentes. Revista da Educação Fisica, 23(3), 399-410.

Guedes, D. P., Neto, J. T. M., Almeida, M. J., Silva, A. J. R. M. (2010). Impacto de fatores sociodemográficos e comportamentais na prevalência de sobrepeso e obesidade de escolares. Revista Brasileira de Cineantropometria e Desempenho Humano, 12(4), 221-231.

Marques, A. T. \& Gaya, A. (1999). Atividade física, aptidão física e educação física para a saúde: Esudos na área pedagógica em Portugal e no Brasil. Revista Paulista de Educação Fisica, 13 (1), 83102.

doi: http://citrus.uspnet.usp.br/eef/uploads/arquivo /v13\%20n1\%20artigo6.pdf

Gaya, A., Lemos, A., Gaya, A., Texeira D., Pinheiro, E., \& Moreira, R. (2016). Manual de testes e avaliação. Projeto Esporte Brasil - PROESP-Br.

Neumark-Sztainer, D., Wall, M. M., Hannan, P. J., Story, M., Croll, J., \& Perry, C. (2003). Correlates of fruit and vegetable intake among adolescents: Findings from Project EAT. Preventive Medicine, 37(3), 198-208. 